\title{
OBESIDADE NA ADOLESCÊNCIA: A INTERDISCIPLINARIDADE COMO ESTRATÉGIA DE PROMOÇÃO DA SAÚDE
}

\author{
Edison Marcos Barreto Filho \\ Instituto Federal de Educação, Ciência e Tecnologia Fluminense, Campos dos Goytacazes, Rio de \\ Janeiro, Brasil
}

\author{
Geilsa Soraia Cavalcanti Valente \\ Universidade Federal Fluminense, Niterói, São Paulo, Rio de Janeiro, Brasil
}

\begin{abstract}
Resumo
O objetivo do estudo foi identificar se docentes de um instituto federal atuam na perspectiva do trabalho interdisciplinar, contribuindo para a promoção da saúde e a prevenção da obesidade de escolares. Para tanto, desenvolveu-se um estudo de caso descritivo, por meio de entrevistas semiestruturadas com 11 docentes, em novembro de 2015, cujos dados foram submetidos à análise de conteúdo. Os resultados apontam para a importância da interdisciplinaridade no contexto do trabalho docente como fator de promoção da saúde e prevenção da obesidade. A escola é um ambiente de discussão, e não se pode achar que uma disciplina ou um docente trabalhando isoladamente pode contribuir para o processo de prevenção da obesidade.
\end{abstract}

Palavras-chave: Comunicação interdisciplinar. Obesidade. Adolescente. Educação Física e treinamento.

\section{Introdução}

A prevalência de excesso de peso na infância e adolescência tem aumentado tanto em países desenvolvidos quanto em países em desenvolvimento, sendo considerada uma pandemia de caráter mundial, impulsionada, em grande medida, pelo crescimento econômico e pela rápida urbanização (SILVA et al., 2014; MALIK; WILLETT; HU, 2013). Esse movimento vem atrelado à modernização e aos avanços tecnológicos que acabam por favorecer a modificação de hábitos de vida dos indivíduos, sobretudo no que se refere à adoção de um estilo de vida sedentário (SILVA; LOPES; SILVA, 2007).

Os comportamentos sedentários envolvem atividades de baixa intensidade com gasto energético reduzido, os quais têm sido associados a outras condutas danosas à saúde das crianças e dos adolescentes, como ingestão de alimentos de alta densidade, uso de bebidas alcóolicas e tabagismo, contribuindo para o aumento de peso e a obesidade entre eles (DIAS et al., 2014).

A obesidade sempre foi alvo de muita discussão e ganha cada vez mais destaque como fator responsável pelo surgimento e pelo agravamento de doenças crônicas como hipertensão e diabetes, depressão e redução da qualidade de vida (LEAL et al., 2012). 
A nutrição desequilibrada e a falta de exercícios físicos são fatores determinantes para a obesidade na adolescência. Assim, é correto dizer que a alteração nos costumes alimentares e de vida sedentária dos adolescentes, visando a hábitos salutares, propõe uma vida mais conveniente em termos de qualidade de vida. Nesse sentido, a educação física voltada para a prevenção é, sem dúvida, a grande contribuição que a educação nos dias atuais pode oferecer à sociedade e aos jovens estudantes, no que se refere à prevenção de enfermidades relacionadas à obesidade.

As atividades físicas são importantes na diminuição do peso corporal, já que estabelecem diminuição do lipídio corporal. A conservação ou o aumento do tecido magro mantém um alto nível de metabolismo de repouso (basal), pois o peso isento de gordura continua sendo metabolicamente mais ativo que a gordura corporal. Isso reduz a tendência do corpo em armazenar calorias, aumentando a eficácia potencial do programa de redução ponderal (MCARDLE et al., 2011).

Assim, aconselha-se que a educação física tenha regularidade e sequência, e que não sofra interrupções, a fim de que as crianças e adolescentes tenham um desenvolvimento crescente em sua estrutura física, compatível com sua idade, peso e altura.

A prática regular de atividade física é um dos fatores que pode estar inversamente associado ao tempo de atividades sedentárias (MALIK; WILLETT; HU, 2013); entretanto, autores acreditam que o declínio de uma não promove a elevação da outra (ESTIMA; COSTA; SICHIERI, 2009; DIAS et al., 2014).

Nesse sentido, a prevenção da obesidade não se arraiga apenas na prática de atividades físicas regulares, pois trata-se de um fenômeno multifatorial, exigindo, portanto, uma abordagem interdisciplinar. Para Ferreira (2011), a interdisciplinaridade não minimiza tampouco neutraliza as disciplinas, ao contrário, propõe que uma sirva de apoio para a construção, análise e interpretação de diferentes conhecimentos, configurando-se como eixo integrador.

Godoy (2014) infere que o trabalho interdisciplinar do educador é um trabalho profilático, que pode prevenir doenças psíquicas e trabalhos de reeducação. Ressalta-se, no entanto, que, apesar da importância metodológica do trabalho interdisciplinar, não se pode fazer dele um fim, mas uma estratégia para o estabelecimento de uma nova pedagogia, a da comunicação (FERREIRA, 2011).

Nessa perspectiva, o Projeto Pedagógico Institucional (PPI) do Instituto Federal de Educação, Ciência e Tecnologia Fluminense (IFF) assume a interdisciplinaridade como uma das ferramentas pedagógicas que enfatiza metas que possam contribuir para a práxis pedagógica da comunidade acadêmica, com a construção, (re)construção e produção do conhecimento pelos sujeitos desse processo. Assim, a Educação Física deveria assumir o papel de orientadora na construção de um programa interdisciplinar participativo de prevenção da obesidade na escola, que inclua docentes, familiares e outros profissionais.

Nessa vertente, o IFF, por meio de sua política institucional de apoio à extensão, desenvolve projetos de responsabilidade social, que objetivam atender alunos encaminhados pelo Serviço Médico e Odontológico do IFF e realizar trabalhos de prevenção nesta área, atendendo, portanto, ao Decreto $\mathrm{n}^{\circ}$ 6.286, de 5 de dezembro de 2007, que institui o Programa Saúde na Escola (PSE) como agente de promoção da saúde e sendo fundamental para o desenvolvimento de ações coletivas e interdisciplinares de saúde (BRASIL, 2007).

Diante do exposto, este estudo objetivou identificar se docentes do IFF atuam na perspectiva do trabalho interdisciplinar, contribuindo para a promoção da saúde e a prevenção da obesidade de escolares. 


\section{Metodologia}

Trata-se de pesquisa de natureza qualitativa, descritiva, do tipo estudo de caso, desenvolvida na unidade de Educação Física do IFF, do campus Campos Centro, localizada no município de Campos dos Goytacazes (RJ).

A população foi constituída pelos professores do IFF que desenvolvem atividades com escolares do Ensino Médio Integrado. Para determinação da amostra, se consideraram os seguintes critérios de inclusão: ser professor do quadro permanente do curso e desenvolver atividades regulares no cenário do estudo, em disciplina de Educação Física para escolares. Como critério de exclusão, definiu-se: estar em férias ou licença no período definido para a coleta de dados, qual seja: novembro de 2015. De tal modo, a amostra final foi constituída por 11 docentes.

Como técnica de coleta de dados, utilizou-se a entrevista semiestruturada guiada por roteiro, cujas questões referiam-se ao perfil do respondente, a sua formação e experiência profissional, e opinativas relacionadas ao objeto de estudo. As entrevistas foram gravadas em equipamento do tipo $\mathrm{mp} 3$, posteriormente transcritas integralmente e tratadas por análise temática de conteúdo, segundo Bardin (2011).

A análise foi operacionalizada por meio de três fases: organização do material e constituição do corpus de análise; destacamento dos núcleos de sentido que compuseram a comunicação dos depoentes e cuja presença ou frequência de aparição deu significado para o objeto de estudo; e categorização com amparo no referencial teórico de Ivani Fazenda, no que diz respeito à interdisciplinaridade na educação. Desse processo; resultaram quatro categorias: a) A interdisciplinaridade na percepção e no trabalho dos docentes; b) Obesidade e promoção da saúde na escola: um olhar crítico-reflexivo; c) Estratégias de educação permanente, tendo em vista a prevenção da obesidade; d) A interdisciplinaridade como fator de promoção da saúde e prevenção da obesidade no IFF.

Em atendimento aos preceitos éticos estabelecidos na Resolução no 466/12, o projeto de pesquisa foi enviado ao Comitê de Ética em Pesquisa do Hospital Universitário Antônio Pedro, da Universidade Federal Fluminense (HUAP/UFF), tendo sido aprovado pelo Parecer $\mathrm{n}^{\mathrm{o}}$ 1.269.415. Todos os participantes assinaram o Termo de Consentimento Livre e Esclarecido e tiveram suas identidades preservadas. Para a identificação dos sujeitos, utilizaram-se nome de cores escolhidas aleatoriamente.

\section{Resultados e discussão}

\section{Perfil dos respondentes}

Constatou-se uma maioria absoluta do sexo masculino entre os respondentes $(n=10)$, provavelmente em decorrência das dificuldades que as mulheres tinham de exercer profissões que guardavam prevalência masculina, como a Educação Física. Esse cenário só começa a se alterar a partir da segunda metade do século XX, sobretudo pós Revolução Feminista (FERRETI, 2011).

A maioria dos docentes cursou pós-graduação stricto sensu (dois doutores; quatro mestres; quatro mestrandos e um especialista); a média de idade foi de 52,7 anos, e o tempo médio de trabalho no IFF de 20,6 \pm 5,9 anos. Estes dados indicam a experiência do grupo e, neste particular, com a instituição, o que poderia contribuir para o desenvolvimento de ações ligadas à interdisciplinaridade dentro da instituição. 


\section{A interdisciplinaridade na percepção e no trabalho dos docentes}

Os docentes afirmaram conhecer os conceitos de transdisciplinaridade, multidisciplinaridade e interdisciplinaridade, no entanto, percebeu-se dúvida e imprecisão ao definirem esses conceitos:

[...] interdisciplinaridade tenta agregar várias disciplinas com o mesmo objetivo comum com cada uma delas [...]. Multidisciplinaridade, termo novo, acho que seria mais disciplinas trabalhando em conjunto [...]. Transdisciplinaridade desconheço [...] (Cinza).

[...] vínculo, adequação de conteúdos e objetivos [...] (Branco).

Uma formação integrada e articulada nas diversas áreas do conhecimento requer dos educadores abertura para buscar a construção de uma proposta curricular contextualizada com a realidade escolar, na perspectiva de formar cidadãos comprometidos com a transformação social de sua comunidade. Essa integração, no entanto, não pode ser pensada apenas no nível de conteúdos ou métodos, pois se corre o risco de deturpar a ideia primeira da interdisciplinaridade, que é a formação global (FERREIRA, 2011).

Questionados se trabalham dentro da lógica interdisciplinar, alguns depoentes afirmaram que sim, ao passo que outros consideraram que não atuam nessa perspectiva; isso denota que o movimento de atuar conforme as diretrizes do PPI do IFF ainda é individual, por iniciativa pessoal.

\section{[...] Infelizmente no dia a dia não trabalhamos [...] (Cinza). \\ $[\ldots]$ trabalho com a interdisciplinaridade [...] (Branco).}

Fazenda (2010) já demonstrava a importância de formar docentes para o trabalho interdisciplinar, em um processo paralelo ao desenvolvimento de pesquisas teóricas na área, visando subsidiar a atuação dos professores para que a relação entre teoria e prática seja continuamente aperfeiçoada.

O pensar interdisciplinar tem como um dos fundamentos a ideia de que nenhum conhecimento é autossuficiente. Isto significa dizer que há a necessidade de diálogo entre as diferentes fontes do saber (FAZENDA, 2010). Nesse aspecto, questionou-se se há discussões e incentivo para o trabalho na perspectiva da interdisciplinaridade no âmbito do IFF:

\section{[...] Não. [...] comodismo, não querem sair da zona de conforto (Amarelo). \\ [...] Não. [...] Somente nas reuniões pedagógicas iniciais (Cinza).}

Até é, mas não temos contato com outras disciplinas importantes dentro da escola (Rosa).

Percebe-se a necessidade de maior atuação da coordenação do IFF para auxiliar na integração das disciplinas e para incentivar o trabalho na lógica interdisciplinar. Essa abordagem referenda uma prática na qual o sujeito percebe a necessidade de estabelecer relações entre os conteúdos abordados nas ambiências dos discentes, na compreensão de um dado fenômeno ou na resolução de determinado problema, conforme se propõe no PPI do IFF.

Nesse aspecto, o PPI compreende o educador como sujeito de sua ação pedagógica, capaz de elaborar programas e métodos de ensino-aprendizagem, sendo competente para inse- 
rir sua escola numa comunidade. Deduz-se, assim, que a ação pedagógica por meio da interdisciplinaridade aponta para a construção de uma escola participativa na formação do sujeito social (GADOTTI, 1996).

Infere-se, portanto, que sendo o discente o grande beneficiário deste processo, o educador não pode desconhecer as ações pedagógicas, sob pena de oferecer uma educação fragmentada, que possivelmente não irá atender aos anseios da sociedade.

\section{Obesidade e promoção da saúde na escola: um olhar crítico-reflexivo}

Segundo dados do Ministério da Saúde (BRASIL, 2015), 52,5\% dos brasileiros estão acima do peso e $17,9 \%$ da população está obesa. A prevalência desses fenômenos é menor entre os jovens, apesar disso, o índice é preocupante. Entre 2008 e 2009, 21,5\% e 5,8\% da população com idade entre 10 e 19 anos estavam com excesso de peso e obesidade, respectivamente (IBGE, 2010).

Uma das morbidades mais imediatas da obesidade é o diabetes, e há evidências de que sua prevalência dobrará até 2030 nas Américas. A projeção da tendência, observada em anos recentes, de aumento da prevalência de obesidade faz prever que em pouco mais de uma década atingiremos níveis próximos aos atualmente observados nos Estados Unidos (SCHMIDT et al., 2011).

Nesse contexto, entende-se que a escola é um ambiente essencial para promover intervenções que possam contribuir para a melhoria desse quadro, o que ratifica ainda mais a importância de se promover saúde dentro do ambiente escolar como forma de elucidar fatos determinantes para a qualidade de vida da população, buscando hábitos saudáveis por meio do trabalho interdisciplinar.

Sobre a Educação Física escolar e a promoção da saúde, verificou-se que os docentes do IFF acreditam que sua disciplina é fundamental para a prevenção da obesidade. No entanto, afirmaram que esta prática ainda carece de maiores esforços no âmbito pedagógico:

[...] o docente tem a percepção de que tão somente sua disciplina pode ser uma aliada na prevenção de doenças relacionadas à obesidade, porém não fazemos corretamente [...] (Bege).

[...] a disciplina já contribui mesmo não sendo de forma interdisciplinar [...] objetivo principal é a manutenção da saúde [...] (Cinza).

$[\ldots]$ informar e alertar nossos alunos destes e demais problemas relacionados à manutenção da saúde já é suficiente [...] (Branco).

Quando perguntados a respeito do planejamento de suas aulas ou sobre questões voltadas para a prática preventiva no que se refere à obesidade, três docentes afirmaram que nunca realizaram este tipo de trabalho. Nesta ótica, acredita-se que a interdisciplinaridade pode possibilitar a ampliação dos resultados da prática pedagógica em saúde. Porém, não existe suporte para que ela seja aplicada no processo de trabalho do docente, que também não sabe como desenvolvê-la, apesar de se tratar de um princípio norteador para a prática acadêmica explicitado no PPI da instituição.

O pensar interdisciplinar, de acordo com Ferreira (2011, p.10), tem como um dos fundamentos a ideia de que nenhum conhecimento é autossuficiente, havendo a necessidade de diálogo entre as fontes de saber. Tal aspecto encontra-se permeado em uma das diretrizes que retrata a atitude dinâmica do currículo no desenvolvimento da ação pedagógica ou de abordagem aplicativa das áreas do conhecimento, a qual implica estabelecer articulações e interações que sejam pertinentes e adequadas à produção do conhecimento dos sujeitos. Assim, tendo em 
vista que a interdisciplinaridade oportuniza a integração e a articulação do currículo, provocando intercâmbios reais, evidencia-se a relevância de envidar esforços para a implementação de um projeto interdisciplinar.

Observou-se uma preocupação em se buscar informações referentes à obesidade e a sua prevenção. Entretanto, a aplicação de determinadas ações ou conteúdos depende também da iniciativa de cada docente:

[...] Sempre houve uma preocupação muito grande, mas eu não procuro aqui dentro do IFF [...] (Rosa).

[...] É fundamental, temos que estar embasados para discutir com nossos alunos sobre o assunto [...] (Amarelo).

Constatou-se falta de integração e articulação curricular, o que prejudica ações interdisciplinares. Também ficou claro que o desconhecimento por grande parte dos docentes sobre o PSE é um fator preocupante (7 dos 11 não conhecem o programa), uma vez que este prima pela promoção da saúde dentro do ambiente escolar. Ressalta-se o fato de que as informações adquiridas e aplicadas não seguem um padrão preestabelecido nas reuniões pedagógicas, o que ocasiona, assim, divergências entre muitos pontos das práticas pedagógicas, tornando o processo de ensino fragmentado.

$[\ldots]$ na escola não temos tempo suficiente para informar o aluno com clareza sobre a obesidade [...] (Rosa).

[...] O problema não se restringe apenas a nossa atuação; poderíamos colaborar com o processo, mas vejo como um problema mais amplo [...] (Amarelo).

Os depoimentos acima denotam a importância da realização do trabalho interdisciplinar, tendo em vista a complementaridade de informações possíveis de serem implementadas por meio desta prática, pois o educador físico sozinho não consegue efetivar, apenas durante as suas aulas, a amplitude de conhecimentos necessários para os adolescentes. É preciso, inclusive, incluir a família nesse processo:

[...] não depende da instituição escolar e sim familiar [...] na escola faz uma coisa e em casa não tem como acompanhar [...] (Laranja).

Sobre os modos de contribuição da Educação Física e da prática pedagógica para a prevenção da obesidade, tomando por base a realidade por eles vivida, os docentes responderam:

[...] A disciplina contribui a partir do momento que passa a informação do que é obesidade, de como é que se alimenta, de como é o gasto calórico, como é o acúmulo de energia, na verdade é informação, mas nem sempre quem tem as informações as utiliza (Marrom).

[...] Promover a prática de atividade física regular que é um componente importante para o controle da obesidade, acho que esta é a melhor forma, além de conteúdos teóricos que possam corroborar para esse controle [...] (Amarelo).

[...] Não sei se haveria uma melhoria, mas eu acho que a conscientização vem da busca do significado; quando você compreende cognitivamente o que está 
acontecendo ao seu redor, seu corpo de maneira geral e com a sociedade, você começa a se questionar no seu dia a dia e isso traz uma melhora. Agora, há uma quantidade de coisas que interferem na adesão de um programa de atividade física [...] (Branco).

Por meio destas respostas, fica evidenciado que os docentes têm consciência dos aspectos inerentes à sua prática, enfatizando que a informação aliada à prática da atividade física são ferramentas essenciais para a prevenção e/ou promoção da saúde dos alunos.

\section{Estratégias de educação permanente para a prevenção da obesidade}

A educação permanente em saúde pode corresponder à educação em serviço, quando esta coloca a pertinência dos conteúdos e instrumentos para a formação técnica submetida a um projeto de mudanças institucionais ou políticas das ações. Pode corresponder à educação continuada quando esta pertence à construção objetiva de quadros institucionais e à investidura de carreiras por serviço. Pode, também, corresponder à educação formal de profissionais, quando esta se apresenta aberta às multiplicidades da realidade de vivências e se coloca em aliança de projetos integrados entre o trabalho e o ensino (CECCIM, 2005).

Sobre esse assunto, quatro docentes relataram que, durante sua formação acadêmica, não cursaram nenhuma disciplina voltada para a área de saúde. Ao serem indagados sobre se buscam por capacitação e informações referentes à prevenção da obesidade, as respostas variaram:

\section{[...] Sim [...] eventos, internet, palestras (Laranja) \\ [...] Sim [...] palestras, informações diárias, contato com exames (Preto). \\ [...] Não [...] (Azul; Roxo; Marrom). \\ [...] Sim [...] pós-graduação em Nutrição (Bege).}

Destaca-se o fato de que um dos depoentes realizou pós-graduação em Nutrição, o que compõe uma importante iniciativa deste profissional no que tange à ampliação do conhecimento relacionado à saúde e que, certamente, deve fazer diferença no seu modo de agir. Se somos atores ativos das cenas de formação e trabalho, os eventos em cena nos produzem diferença, nos modificam, colocando-nos em permanente produção (CECCIM, 2005).

Neste sentido, a interdisciplinaridade no âmbito escolar constitui-se como alternativa metodológica para superar o ensino fragmentado, sendo capaz de potencializar um maior significado aos conteúdos escolares, contribuindo para uma formação integral que prepare para a vida (OLIVEIRA, 2016; RIZZO, 2016).

A interdisciplinaridade escolar tem como principais finalidades a difusão de conhecimentos e a formação, dependendo da organização dos conhecimentos escolares sobre os planos curriculares, didáticos e pedagógicos. Sendo assim, para realizar este trabalho, deve-se partir do ponto inicial, por meio da educação permanente dos docentes. Desta maneira, estas informações poderiam ser trabalhadas com eles, para que possam ser multiplicadores da prática educativa de forma interdisciplinar.

Torna-se importante que haja uma reflexão no que tange à forma de explorar as ações interdisciplinares de maneira organizada e orientada. Para tanto, faz-se necessário mobilizar esforços por meio da coordenação de área no sentido de aplicar mecanismos que possam colaborar para a participação dos docentes, a partir da criação de um trabalho voltado para a implementação de um programa interdisciplinar, no qual serão discutidas e instituídas as principais ações para a prevenção da obesidade e a promoção da saúde no cenário pesquisado. 
Ressalta-se que, durante as entrevistas, os profissionais servidores da área de nutrição, psicologia e médica do instituto não foram mencionados como forma de apoio, ou fontes de informações acerca da prevenção da obesidade.

\section{A interdisciplinaridade como fator de promoção da saúde e prevenção da obesidade no IFF}

Este estudo também buscou verificar de que forma o trabalho interdisciplinar dos docentes poderia refletir-se em uma ação de prevenção da obesidade e promoção da saúde na escola. Vale destacar, pela análise das entrevistas, que existe, para grande parte dos docentes, a preocupação em permanecer em constante capacitação. Por outro lado, não há uma aplicação desses níveis de conhecimento na prática cotidiana de forma interdisciplinar, como relatam muitos docentes, bem como estão ausentes ou insuficientes as discussões com a coordenação de área. Outro ponto que merece destaque foi a resposta de um dos docentes que vincula a promoção da saúde a um benefício estético, conforme o depoimento a seguir:

[...] Através das práticas e das relações, informações sobre alimentos, melhoria da capacidade física e principalmente estética [...] (Preto).

Outros entrevistados evidenciam a importância da interdisciplinaridade quando relatam que:

[...] a atividade física hoje é uma grande ferramenta para o controle e prevenção da obesidade, [...] se minha disciplina fosse aliada a outra que abordasse esse tema, teríamos condições de realizarmos um bom trabalho [...] (Bege).

[...] a obesidade é multifatorial, ela não é decorrente apenas do sedentarismo, tem o problema da alimentação e sedentarismo. Daí, teríamos que trabalhar com as pessoas da área de informática, pensar sobre a questão de ficar muito tempo na frente do computador ou videogame, por isso ela é multifatorial e por isso deveria ter um conjunto de pessoas de várias disciplinas conversando para que tudo fosse solucionado. E é difícil fazer isso na escola hoje, ainda [...] (Rosa).

Indagados a respeito da realização de um trabalho interdisciplinar numa proposta de melhoria na ação de prevenção a obesidade e de que forma sua disciplina poderia contribuir, os docentes responderam:

[...] com a prática regular, abordando temas teóricos [...] (Vermelho).

[...] acredito que através da parte de alimentação e o questionamento sobre eles e também sobre atividade física em si [...] (Azul).

Sim. [...] Palestras, seminários e práticas desportivas (Bege).

Ainda sobre esse aspecto, o depoimento a seguir evidencia que existe a necessidade de envolver o aluno como protagonista do processo, lançando-se mão de outras formas de educar:

[...] Poderia ter um envolvimento maior dos alunos através de palestras, aulas de campo, experiências práticas, algumas coisas que pudessem motivar o aluno [...] ter conhecimento não só da educação física, mas das coisas que a cer- 
cam $[\ldots]($ Roxo).

Refletindo sobre o exposto, corrobora-se que é preciso ultrapassar as fronteiras dos conhecimentos que pretendem traduzir a verdade da ciência para a aprendizagem na dimensão micropolítica do trabalho, condição que somente se expressa em ato e acumula na ordem das aprendizagens como sensação, afeto e afetação. A complexidade dessa ordem de aprendizagens dá o sentido da proposição da educação na saúde como novo domínio de conhecimentos, atravessado pela educação e pela cidadania (CECCIM; FERLA, 2009).

O papel das práticas educativas deve ser crítico e incisivamente revisto para que almeje a possibilidade de pertencer aos serviços/profissionais/estudantes a que se dirigem, de forma que os conhecimentos que veiculam alcancem significativo cruzamento entre os saberes formais previstos pelos estudiosos ou especialistas e os saberes operadores das realidades detidos pelos profissionais em atuação - para que viabilizem autoanálise e principalmente autogestão (CECCIM, 2005).

Desta forma, os saberes formais devem estar implicados com movimentos de autoanálise e autogestão dos coletivos da realidade, pois são os atores do cotidiano que devem ser protagonistas da mudança de realidade desejada pelas práticas educativas (CECCIM, 2005).

Ao analisar a promoção da saúde e da prevenção da obesidade, não se deve subjugar os fatos reais de que a obesidade é uma doença grave, tampouco achar que é um problema individual, alimentar ou genético. A escola é um ambiente de discussão, e não se pode achar que uma disciplina ou um docente trabalhando isoladamente pode contribuir para o processo de prevenção da obesidade.

A criatividade, a inovação, a integração e a interação são componentes essenciais para criação do produto e, para que isso ocorra, sugiro como primeiro passo nos atentar para a importância de romper paradigmas e utilizar como aporte os quatro fundamentos citados por Fazenda (2010), devendo-se compreender a importância do movimento dialético; "nunca devemos desprezar as experiências vividas - elas constituem-se na possibilidade da inovação, da revisão e da análise interdisciplinar" (FAZENDA, 2010).

Para implementar um trabalho desta grandeza, faz-se necessária a participação de profissionais dispostos a reverter este grave problema de saúde, que já é considerado uma epidemia. Será por meio de pequenas iniciativas que poderemos transformar algumas atitudes, fazendo a integração do trabalho interdisciplinar, de servidores administrativos e docentes com a participação dos pais, familiares ou responsáveis em prol da saúde dos alunos. Daí a tamanha importância para a educação permanente em saúde que tem uma proposta políticopedagógica e mantém o cotidiano do trabalho ou da formação em constante análise, construindo espaços coletivos para a reflexão e a avaliação de sentido dos atos produzidos no cotidiano.

\section{Conclusão}

Considera-se que o momento em que vivemos na educação nacional é repleto de imensas transformações que, na maioria das vezes, vão além da compreensão de todos os envolvidos (professor, aluno, pais de alunos, diretores, coordenadores). Portanto, este trabalho sugere que possamos estar sempre atentos às questões abordadas, principalmente no que diz respeito à coletividade para que ações de promoção da saúde ocorram, acompanhadas de um trabalho interdisciplinar com o objetivo de prevenir a obesidade na adolescência com a criação de um programa escolar interdisciplinar dentro dos institutos federais.

No instituto federal, cenário deste estudo, constatou-se que alguns professores atuam na perspectiva interdisciplinar, mas ainda se carece de maior integração entre as disciplinas e 
docentes para que as estratégias educativas sejam eficazes e atuem como fator de promoção da saúde e de prevenção da obesidade entre os estudantes.

Os profissionais de Educação Física, tendo em vista os novos caminhos pelos quais a sociedade avança, necessitam reconhecer o novo paradigma com vistas a adotar um posicionamento crítico em relação aos novos padrões. Abordar tais questões significa, dentro da escola, assumir posicionamentos que façam das aulas e da formação momentos prazerosos, nos quais os alunos se sintam motivados para vivenciar este processo de formação. Propor uma intervenção educacional, neste sentido, significa, num primeiro momento, identificar de que forma a Educação Física poderia contribuir para a promoção da saúde e o controle da obesidade na adolescência. Assim, deve-se reavaliar a importância de se ampliar o desenvolvimento das pesquisas teóricas na área, visando subsidiar a atuação dos professores, possibilitando a continuidade e o aperfeiçoamento no processo de interação entre a teoria e a prática.

\title{
OBESITY IN ADOLESCENCE: INTERDISCIPLINARITY AS HEALTH PROMO- TION STRATEGY
}

\begin{abstract}
The aim of the study was to identify whether teachers of a federal institute works in an interdisciplinary perspective, contributing to the promotion of health and prevention of obesity among students. Therefore, we developed a descriptive case study, through semi-structured interviews with eleven teachers, in November 2015, whose data were subjected to content analysis. The results point to the importance of interdisciplinarity in the context of teaching as a factor in promotion and prevention of obesity. The school is a discussion environment, and you cannot think that a subject or a teacher working in isolation can contribute to the process of preventing obesity.
\end{abstract}

Keywords: Interdisciplinary communication. Obesity. Adolescent. Physical Education and training.

\section{LA OBESIDAD EN LA ADOLESCENCIA: LA INTERDISCIPLINARIEDAD COMO ESTRATEGIA DE PROMOCIÓN DE LA SALUD}

\section{Resumen}

El objetivo del estudio fue identificar si los profesores de un instituto federal trabajan dentro de una perspectiva interdisciplinaria que contribuya para la mejoría de la salud y prevención de la obesidad entre los estudiantes. Para eso, se desarrolló un estudio de caso descriptivo, a través de entrevistas semiestructuradas con once profesores, en noviembre de 2015, cuyos datos fueron sometidos al análisis de contenido. Los resultados orientan hacia la importancia de la interdisciplinariedad en el contexto del trabajo docente como un factor en la mejoría y prevención de la obesidad. La escuela es un ambiente de discusión, y no se puede pensar que una asignatura o un maestro que trabaja de forma aislada pueden contribuir al proceso de prevención de la obesidad.

Palabras clave: Comunicación interdisciplinaria. Obesidad. Adolescente. Educación Física y Entrenamiento.

\section{Referências}

BARDIN, L.. Análise de conteúdo. São Paulo: Edições 70, 2011. 229 p. 
BRASIL. Decreto no 6.286, de 5 de dezembro de 2007. Institui o Programa Saúde na Escola (PSE), e dá outras providências. Diário Oficial [da] República Federativa do Brasil, Brasília, p. 2, 6 dez. 2007. Seção 1.

BRASIL. Ministério da Saúde. Secretaria de Vigilância em Saúde. Departamento de Vigilância de Doenças e Agravos não Transmissíveis e Promoção da Saúde. Vigitel Brasil 2014: Vigilância de fatores de risco e proteção para doenças crônicas por inquérito telefônico. Brasília: Ministério da Saúde, 2015.

CECCIM, R. B. Educação Permanente em Saúde: desafio ambicioso e necessário. Interface Comunicação, Saúde, Educação, Botucatu, v. 9, n. 16, p. 161-177, set. 2004/fev. 2005, 2005.

CECCIM, R. B.; FERLA, A. A. A educação e saúde: ensino e cidadania como travessia de fronteiras. Trabalho, Educação e Saúde, Rio de Janeiro, v. 6, n. 3, p. 443-456, nov.2008/fev.2009.

DIAS, P. J. P.; DOMINGOS, I. P.; FERREIRA, M. G., et al. Prevalência e fatores associados aos comportamentos sedentários em adolescentes. Revista de Saúde Pública, São Paulo, v. 48, n. 2, p. 266-274, abr. 2014.

ESTIMA, C. C. P.; COSTA, R. S.; SICHIERI, R. et al. Meal consumption patterns and anthropometric measurements in adolescents from a low socioeconomic neighborhood in the metropolitan area of Rio de Janeiro, Brazil. Appetite, v. 52, n. 3, p. 735-739, jun. 2009.

FAZENDA, I. C. A. Interdisciplinaridade: história, teoria e pesquisa. Campinas, SP: Papirus, 2010.

FERREIRA, S. L. Introduzindo a noção de interdisciplinaridade. In: FAZENDA, I. C. A. (Org.) Práticas interdisciplinares na escola. 12 ed. São Paulo: Cortez, 2011.

FERRETI, M. A. C. A formação da lutadora: estudo sobre mulheres que praticam modalidades de luta. 2011. 111 f. Dissertação (Mestrado em Educação Física) - Escola de Educação Física e Esporte, Universidade de São Paulo, São Paulo, 2011.

GADOTTI, M. Educação contra educação. 5 ed. São Paulo. Paz e Terra, 1996.

GODOY, H. P. Consciência espiritual. In: FAZENDA, I. C. A.; GODOY, H. P. (Org.). Interdisciplinaridade: pensar, pesquisar, intervir. São Paulo: Cortez, 2014.

INSTITUTO BRASILEIRO DE GEOGRAFIA E ESTATÍSTICA (IBGE). Pesquisa de orçamentos familiares 2008-2009: antropometria e estado nutricional de crianças, adolescentes e adultos no Brasil. Rio de Janeiro: IBGE, 2010.

LEAL, V. S.; LIRA, P. I. C.; OLIVEIRA, J. S.; MENEZES, R. C. E. de; SEQUEIRA, L. A. S.; ARRUDA NETO, M. A.; ANDRADE, S. L. L. S. de; BATISTA FILHO, M. Excesso de peso em crianças e adolescentes no Estado de Pernambuco, Brasil: prevalência e determinantes. Caderno de Saúde Pública, Rio de Janeiro, v. 28, n. 6, p. 1175-1182, jun. 2012. 
MALIK, V. S.; WILLETT, W. C.; HU, F. B. Global obesity: trends, risk factors and policy implications. Nature Reviews Endocrinology, London, v. 9, n. 1, p. 13-27, jan. 2013.

MCARDLE, W. D; KATCH, F. I; KATCH, V. L. Fundamentos de fisiologia do exercício. 7 ed., Rio de Janeiro: Guanabara Koogan, 2011.

OLIVEIRA, E. B.; MANSO, M. H. S.; SANTOS, F. N. S. et al. Os desafios da interdisciplinaridade no currículo da área das ciências da natureza de uma escola estadual. Interdisciplinaridade, São Paulo, n. 8, abr. p. 31-47, 2016.

RIZZO, D. T. S.; ARANHA, A. C. M.; FREITAS, C. M. S. M.; DAOLIO, J.; LOPES, J. C. Educação física escolar e esporte: significações de alunos e atletas. Pensar a Prática, Goiânia, v. 19, n. 2, abr./jun. 2016.

SCHMIDT, M. I.; DUNCAN, B. B.; SILVA, G. A.; MENEZES, A. M; MONTEIRO, C. A.; BARRETO, S. M.; CHOR, D.; MENEZES, P. R. Chronic non-communicable diseases in Brazil: burden and current challenges. The Lancet, New York, v. 377, n. 9781, p. 1949-61, jun. 2011.

SILVA, K. S.; LOPES, A. S.; SILVA, F. M. Comportamentos sedentários associados ao excesso de peso corporal. Revista Brasileira de Educação Física Esportiva, São Paulo, v. 21, n. 2, p. 135-141, abr./jun. 2007.

SILVA, Y. M. Q. et al. Prevalência de excesso de peso em crianças e adolescentes de um projeto esportivo em Maceió-AL. Revista Brasileira de Ciências da Saúde, v. 18, n. 4 , p. 67-74, 2014.

Recebido em: 22/09/2016

Revisado em: 25/09/2017

Aprovado em: 16/10/2017

Endereço para correspondência:

prof.edisonmarcos@gmail.com

Edison Marcos Barreto Filho

Instituto Federal de Educação, Ciência e Tecnologia Fluminense

Campus Campos Centro

Rua Dr. Siqueira, 273 - Parque Dom Bosco,

Campos dos Goytacazes, RJ

CEP: $28030-130$ 